\title{
A Simple and Robust 40-Gb/s Wavelength Converter Using Fiber Cross-Phase Modulation and Optical Filtering
}

\author{
Bengt-Erik Olsson, Peter Öhlén, Lavanya Rau, and Daniel J. Blumenthal, Senior Member, IEEE
}

\begin{abstract}
In this letter, 40-Gb/s return-to-zero data wavelength conversion is demonstrated using cross-phase modulation in an optical fiber with subsequent conversion of phase modulation to amplitude modulation using an optical filter. The scheme is potentially ultrahigh speed and can be made polarization independent.
\end{abstract}

Index Terms-Cross-phase modulation, optical fiber communication, optical networks, wavelength converters.

\section{INTRODUCTION}

A LL-OPTICAL wavelength conversion can play an important role in future ultrahigh-speed networks due to the significant increase in flexibility and potential reduction in need for optical buffers [1]. Ultrahigh-speed wavelength conversion of return-to-zero (RZ) data has previously been demonstrated using four-wave mixing (FWM) in fiber [2] and semiconductor optical amplifiers (SOA's) [3] and by use of cross-phase modulation (XPM) in the nonlinear optical loop mirror (NOLM) [4]. Using FWM, it is not possible to convert an unknown wavelength to a predetermined wavelength, and the NOLM requires short pulses compared to the bit period and also suffers from stability problems. The wavelength conversion of short pulses has previously been proposed using XPM and soliton formation in a fiber [5] or using fiber XPM and polarization discrimination, i.e., nonlinear polarization rotation [6]. Here, wavelength conversion of $40-\mathrm{Gb} / \mathrm{s}$ data across $8 \mathrm{~nm}$ is demonstrated, and the method will most likely be scalable to much higher bit rate over a broader wavelength range, primarily limited by dispersive walkoff in the fiber. The novel idea here is to utilize XPM in a dispersion shifted fiber (DSF) followed by an optical notch filter. If the incoming data is combined with a continuous-wave (CW) signal and sent through an optical fiber, the data imposes a phase modulation onto the CW light using XPM. This phase modulation generates optical sidebands on the $\mathrm{CW}$ signal, which can be converted to amplitude modulation by suppressing the original $\mathrm{CW}$ carrier using an optical notch filter. The notch filter used here was a loop mirror filter (LMF), which

\footnotetext{
Manuscript received November 30, 1999; revised March 14, 2000. This work was supported under the DARPA sponsored Center for Multidiciplinary Optical Switching Technology (F49620-96-1-0349).

B.-E. Olsson, L. Rau, and D. J. Blumenthal are with the Department of Electrical and Computer Engineering, University of California, Santa Barbara, CA 93106 USA.

P. Öhlén is with Department of Electrical and Computer Engineering, University of California, Santa Barbara, CA 93106 USA, on leave from the Royal Institute of Technology, S-164 40 Kista, Sweden.

Publisher Item Identifier S 1041-1135(00)05596-8.
}

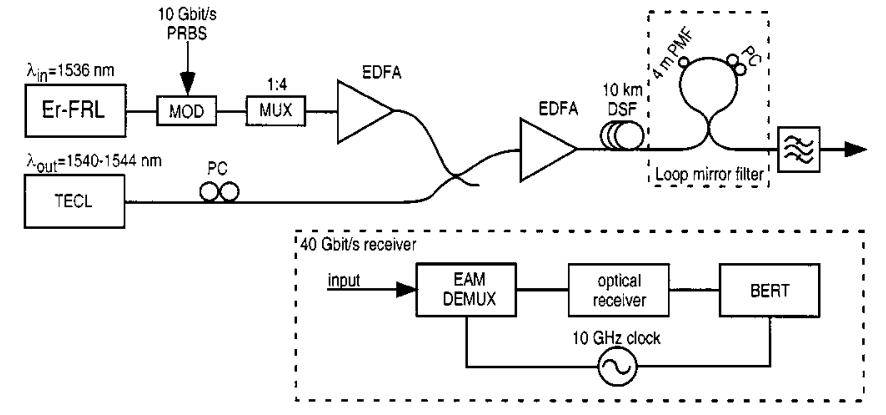

Fig. 1. Experimental setup of the XPM wavelength converter. Er-FRL: fiber ring laser. MOD: LiNbO3 modulator. EDFA: erbium-doped fiber amplifier. PC: polarization controller. PMF: polarization maintaining fiber. EAM: electroabsorption modulator. BERT: bit-error rate test set.

consists of short piece of birefringent fiber in a Sagnac interferometer. Such filter is is tunable and has repetitive notches, which allows conversion to different discrete wavelengths without adjusting the filter. However, in principle, any type of filter can be used, e.g., a fiber Bragg grating.

\section{EXPERIMENTS}

Fig. 1 shows the experimental setup of the wavelength converter. The data pulses were generated with an actively modelocked $10-\mathrm{GHz}$ fiber ring laser giving 10-ps pulses with a timebandwidth product (TBP) of 0.46 at $1536 \mathrm{~nm} .10-\mathrm{Gb} / \mathrm{s}$ pseudorandom bit-stream (PRBS) data $\left(2^{31}-1\right)$ was subsequently encoded and a $40-\mathrm{Gb} / \mathrm{s}$ data stream was obtained by passively multiplexing four times $10-\mathrm{Gb} / \mathrm{s}$ data streams together. The $40-\mathrm{Gb} / \mathrm{s}$ data was combined with $\mathrm{CW}$ light from a tunable external cavity laser and amplified before being injected into a 10-km DSF with a zero dispersion wavelength of $1540 \mathrm{~nm}$. After the DSF, an LMF, consisting of a loop mirror with $4 \mathrm{~m}$ of polarization maintaining fiber and a polarization controller to allow adjustment of the filter wavelength, was used to suppress the original CW light carrier. The transmission function of the filter is shown in Fig. 2. The separation between the notches is $1 \mathrm{~nm}$ (given by the length of the birefringent fiber) and the suppression of the $\mathrm{CW}$ light was better than $27 \mathrm{~dB}$ when adjusting a notch to the wavelength of the $\mathrm{CW}$ light. A subsequent 0.8 -nm bandpass filter was used to select one of the two sidebands and to suppress the original data. The $40-\mathrm{Gb} / \mathrm{s}$ receiver consisted of an electroabsorption modulator (EAM) with 15-ps switch window to demultiplex 40 to $10 \mathrm{~Gb} / \mathrm{s}$.

Fig. 3 shows the spectra, as measured with a spectrum analyzer with $0.1-\AA$ resolution, before and after the LMF. The 


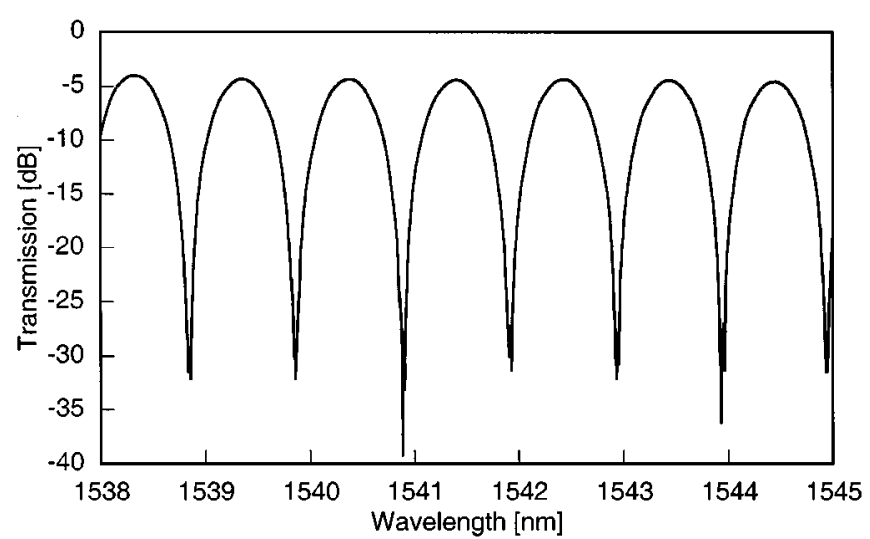

Fig. 2. Transmission through the LMF.

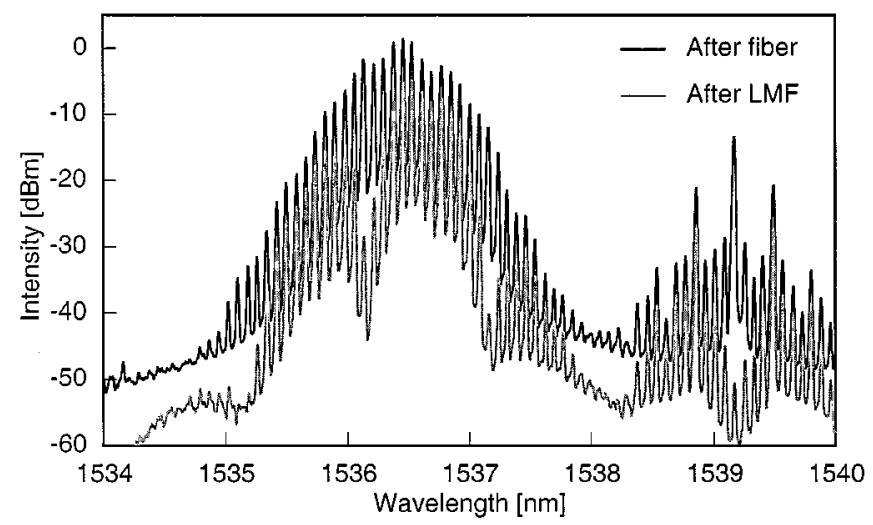

Fig. 3. Output optical spectrum of original data and wavelength converted signal before and after the filter.

original data is spectrally broadened due to self-phase modulation and the carrier at $1539 \mathrm{~nm}$ is suppressed by the LMF, leaving two amplitude modulated sidebands. The longer wavelength sideband originates from the derivative of the leading edge of the input pulse and the sideband toward shorter wavelengths originates from the trailing edge of the pulse. By selecting only one of the sidebands using a bandpass filter with a bandwidth matching the spectral width of the sideband, the output pulsewidth should ideally be shorter than the input pulse due to the derivative origin of the sideband. Due to optical filtering with limited bandwidth and dispersive walkoff between input and output data, the output pulses are slightly broader than the input. The output pulse width was measured as a function of wavelength and, with a 9-ps input pulse, the output pulse width varied from 10 to $12 \mathrm{ps}$ for all output wavelengths from 1538 to $1548 \mathrm{~nm}$. However, the required input peak power for full switching increases with larger conversion span due to the dispersive walkoff. For wavelength conversion from 1536 to $1540 \mathrm{~nm}$, the TBP was 0.46 at both input and output, which indicates the possibility to further transmit the data. Transmission of the wavelength converted pulses through standard fiber showed the same dispersive pulse broadening versus fiber length as 12-ps unchirped Gaussian pulses up to $25 \mathrm{~km}$. The nonlinear transmission characteristic is shown in Fig. 4 for a 10-km DSF as well as for a $5-\mathrm{km}$ DSF as measured with 10-ps input pulses with $10-\mathrm{GHz}$ repetition frequency for wavelength conversion

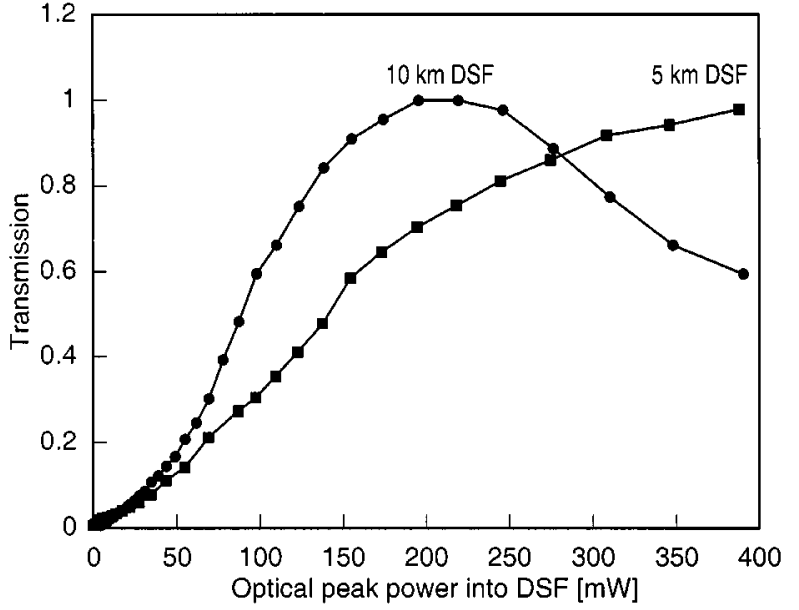

Fig. 4. Transmission versus input peak power for the wavelength converter using a $10-$ and $5-\mathrm{km}$ DSF.

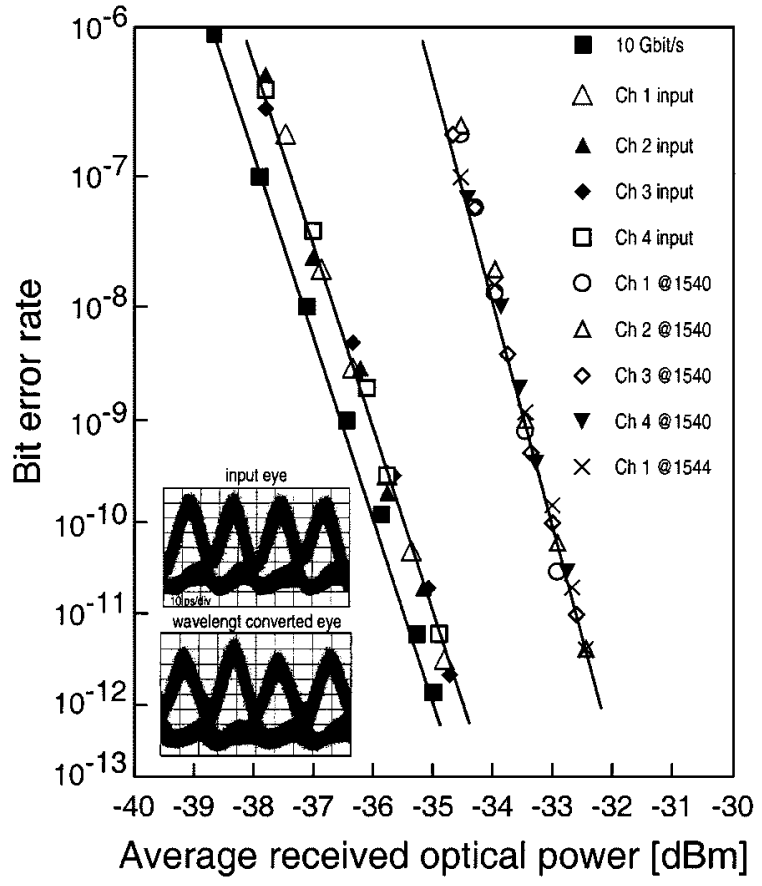

Fig. 5. BER measurements for wavelength conversion of $40-\mathrm{Gb} / \mathrm{s}$ data from 1536 to 1540 and $1544 \mathrm{~nm}$.

from 1536 to $1540 \mathrm{~nm}$. For moderate input power, the transmission follows a $\sin ^{2}$ function due to the transmission function of the LMF, and the transmission function can, to some extent, be determined by the transfer functions of the filters used. With a 10-km DSF, 200-mW peak power was required for full switching and, for 5-km DSF, about $450 \mathrm{~mW}$ was needed. Fig. 5 shows the received eye pattern of the original and wavelength converted $40-\mathrm{Gb} / \mathrm{s}$ data as well as bit-error-rate (BER) measurements for wavelength conversion from 1536 to $1540 \mathrm{~nm}$ and $1544 \mathrm{~nm}$. The power penalty for BER $=10^{-9}$ was $3 \mathrm{~dB}$ compared to the original $10-\mathrm{Gb} / \mathrm{s}$ data and $2.5 \mathrm{~dB}$ compared to the input $40-\mathrm{Gb} / \mathrm{s}$ data for all four $10-\mathrm{Gb} / \mathrm{s}$ channels. This penalty is believed to be partly due to distortion in the LMF and partly due to polarization interference noise between adjacent pulses. Since polarization interleaving was used in the multiplexer, the 
$40-\mathrm{Gb} / \mathrm{s}$ pulse sequence has every other pulse in orthogonal polarization state. Due to polarization dependence in the wavelength converter, any polarization instability in the data pulses will give rise to noise in the converted data. The power penalty for 6-nm wavelength conversion at $10 \mathrm{~Gb} / \mathrm{s}$ was only $0.6 \mathrm{~dB}$ for the same pulsewidth, which indicates that polarization interference might be a major reason. The polarization dependence for XPM in a fiber is $5 \mathrm{~dB}$ and the polarization dependence as measured with a power meter was from 2 to $8 \mathrm{~dB}$ depending on the position and bandwidth of the bandpass filter. However, the polarization dependence can be eliminated by utilizing polarization scrambling [7] or circularly polarized fiber [8]. Even though a $10-\mathrm{km}$ fiber was used, no stability problems were observed, and the system is only mechanically polarization sensitive before combining the data and CW light. Since the LMF was polarization insensitive, environmental disturbances to the $10-\mathrm{km}$ fiber did not affect the performance apart from a small timing shift, which can be compensated for by a clock recovery circuit.

\section{CONCLUSION}

In this letter, a new, simple, and robust wavelength converter is proposed and experimentally demonstrated at $40 \mathrm{~Gb} / \mathrm{s}$. If a fiber with high nonlinearity [9] is utilized together with a high-power erbium-doped fiber amplifier in a polarization independent configuration, the scheme has the potential to realize a compact polarization independent broad-band wavelength converter.

\section{ACKNOWLEDGMENT}

The authors would like to acknowledge V. Kaman and J. Bowers for supplying the EAM.

\section{REFERENCES}

[1] S. L. Danielsen, P. B. Hansen, and K. E. Stubkjaer, "Wavelength conversion in optical packet switching," J. Lightwave Technol., vol. 16, pp. 2095-2108, Dec. 1998.

[2] K. Inoue and H. Toba, "Wavelength conversion experiment using fiber four-wave mixing," IEEE Photon. Technol. Lett., vol. 4, pp. 69-72, Jan. 1992.

[3] S. Murata, A. Tomita, J. Shimizu, and A. Suzuki, "THz optical-frequency conversion of $1 \mathrm{~Gb} / \mathrm{s}$-signals using highly nondegenerate four-wave mixing in an InGaAsP semiconductor laser," IEEE Photon. Tech. Lett., vol. 3, pp. 1021-1023, Nov. 1991.

[4] K. A. Rauschenbach, K. L. Hall, J. C. Livas, and G. Raybon, "All-optical pulse width and wavelength conversion at $10 \mathrm{~Gb} / \mathrm{s}$ using a nonlinear optical loop mirror," IEEE Photon. Technol. Lett., vol. 6, pp. 1130-1132, Sept. 1994.

[5] D. Schadt and B. Jaskorzynska, "Generation of short pulses from CW light by influence of crossphase modulation (CPM) in optical fibers," Electron. Lett., vol. 23, pp. 1090-1091, 1987.

[6] D. M. Patrick and A. D. Ellis, " $10 \mathrm{GHz}$ pulse train derived from a $\mathrm{CW}$ DFB laser using crossphase modulation in an optical fiber," Electron. Lett., vol. 29, pp. 1391-1399, 1993.

[7] B.-E. Olsson and P. A. Andrekson, "Polarization independent all-optical AND-gate using randomly birefringent fiber in a nonlinear optical loop mirror," in Proc. Optical Fiber Comm. Conf., vol. 2, 1998, pp. 375-376.

[8] Y. Liang, J. W. Lou, J. K. Andersen, J. C. Stocker, O. Boyraz, and M. N. Islam, "Polarization-insensitive nonlinear optical loop mirror demultiplexing with twisted fiber," Opt. Lett., vol. 24, pp. 726-728, 1999.

[9] M. Onishi, T. Okuno, T. Kashiwada, S. Ishikawa, N. Akasaka, and M. Nishimura, "Highly nonlinear dispersion-shifted fibers and their application to broadband wavelength converter," Opt. Fiber Technol., vol. 4, pp. 204-214, 1998 